\title{
Análise da geração de energia elétrica por meio da utilização de conversores do tipo TEG em lingotamento contínuo de aços
}

\section{Analyses of the generation of electric energy by using a TEG-type generator during continuous casting of steel}

Rafael Formenton Macedo dos Santos ${ }^{1}$, José Eduardo Spinelli ${ }^{1}$

\author{
${ }^{1}$ Departamento de engenharia de Materiais - DEMa/UFSCar, CP: 13565-905, São Carlos, São Paulo, Brasil. \\ e-mail: formentonrafael@gmail.com; spinelli@ufscar.br
}

\begin{abstract}
RESUMO
A fabricação do aço é um processo que requer enormes quantidades de recursos, como por exemplo combustíveis, minério de ferro, carvão, entre outros. Com a crescente preocupação com o meio ambiente, seja pelas cargas ambientais ou pelo aquecimento global, cresce também a necessidade do desenvolvimento de novas tecnologias que possibilitem um melhor aproveitamento dos recursos disponíveis. Portanto, para as indústrias do aço, torna-se importantíssima a conservação de energia, de forma que a recuperação de calor ganhe destaque. Este trabalho descreve a utilização de um conjunto Thermoelectric Generator (TEG) na recuperação de energia residual. Os módulos TEG, feitos de óxido de manganês e óxido de cálcio, convertem diretamente o calor das placas de aço 1045, ao final do processo de lingotamento contínuo, em energia elétrica. Cada módulo quadrado (lados de $64,5 \mathrm{~mm}$ e espessura de $8,5 \mathrm{~mm}$ ) gera $12,3 \mathrm{~W}$ quando a temperatura do lado quente é de $800^{\circ} \mathrm{C}$ e a do lado frio é de $50^{\circ} \mathrm{C}$. Neste trabalho foi estimada a quantidade de energia possível de recuperar por este conjunto TEG. Para tanto, foram utilizadas simulações matemáticas e métodos de ajuste, avaliando assim os efeitos da temperatura, tempo e da espessura na geração de energia pelo conjunto TEG. Considerando o lingotamento contínuo de placas com $400 \mathrm{~mm}$ de espessura e $1050^{\circ} \mathrm{C}$ de temperatura, lingotada a uma velocidade de $0,3 \mathrm{~m} / \mathrm{min}$, foi estimado um fornecimento de energia elétrica capaz de abastecer 23 residências brasileiras de acordo com a média nacional de consumo.
\end{abstract}

Palavras-chave: TEG. Geração de energia elétrica. Lingotamento continuo. Simulação.

\begin{abstract}
Steel manufacturing is a process that requires large investments in fuels, iron ore, coal, among others. As such, global concerns related to environment preservation increases. As a consequence, alternative technologies may emerge as adequate management platforms of the available resources. For steel industry the conservation of energy has a prime importance, making heat recovery a hot topic. This research work describes the use of a set of Thermoelectric Generator (TEG) in order to recover residual energy during continuous casting operations. The TEG modules are constituted by manganese and calcium oxides, which are able to convert heat from the 1045 carbon steel plates in the end of continuous casting process into electrical energy. Each square module measures $64.5 \mathrm{~mm}$ of side and $8.5 \mathrm{~mm}$ of thickness, being able to generate $12.3 \mathrm{~W}$ if hot side temperature is at $800^{\circ} \mathrm{C}$ and cold side at $50^{\circ} \mathrm{C}$. In the present contribution, estimations of quantity of recovered energy are shown. Thermal simulations using the software Deform as well as fitting methods have been implemented and incorporated to each other so that the effects of temperature, time and plate thickness could be examined. Considering a plate of 1045 steel with $400 \mathrm{~mm}$ of thickness and temperature of $1050^{\circ} \mathrm{C}$, continuous casted at $0.3 \mathrm{~m} / \mathrm{min}$, it was estimated a recovered energy equivalent to the consumption of electrical energy of 23 average Brazilian residences was estimated.
\end{abstract}

Keywords: TEG. Electric power generator. Continuous casting. Simulation. 


\section{INTRODUÇÃO}

A fabricação de aço é um processo que requer enormes quantidades de recursos, dentre eles destacam-se o carvão e minério de ferro, além de grande quantidade de energia na forma de energia elétrica e ou combustíveis. Porém, problemas como o aquecimento global tornaram necessária a otimização do uso dos recursos energéticos, que pode ser possível, por exemplo, através da máxima utilização de tecnologias inovadoras. Desta forma, o desenvolvimento técnico e a introdução de equipamentos que consigam poupar energia, prevenindo o aquecimento global e reduzindo as cargas ambientais, no caso de um dos processos de fabricação de aço, são de extrema importância [1-2].

Uma das possíveis abordagens a esse problema é a recuperação de calor. Portanto a geração termelétrica (Thermoeletric Generator - TEG) adquire uma grande importância, uma vez que esses dispositivos podem converter o calor diretamente em eletricidade. Dentre as características que o tornam uma tecnologia atraente, podemos citar a ausência de peças mecânicas móveis, vida útil longa, não emitem subprodutos nocivos ao meio ambiente durante sua operação e podem ser utilizadas em uma ampla faixa de temperatura [13].

Conversão termoelétrica, além de ocorrer em termopares simples, constitui a base de módulos termoelétricos reversíveis (TEM - Thermoelectric Modules), que podem trabalhar tanto como coolers (Thermoelectric Coller - TEC), quando alimentado com energia elétrica, utilizando-se do efeito de Peltier, ou como geradores (Thermoelectric Generator - TEG), quando alimentado com calor, utilizando-se do efeito de Seedbeck [3-5].

O TEM consiste em pares de termo elementos, compostos de uma junção de semicondutor do tipo $\mathrm{n}$ e do tipo p (junção P-N), dispostos alternadamente que são conectados eletricamente em série com tiras de metal de ligação e encapsulados entre duas placas de cerâmica. Estas placas são eletricamente isolantes, mas termicamente condutoras [5]. A Figura 1, a seguir, é uma representação esquemática de um TEG.

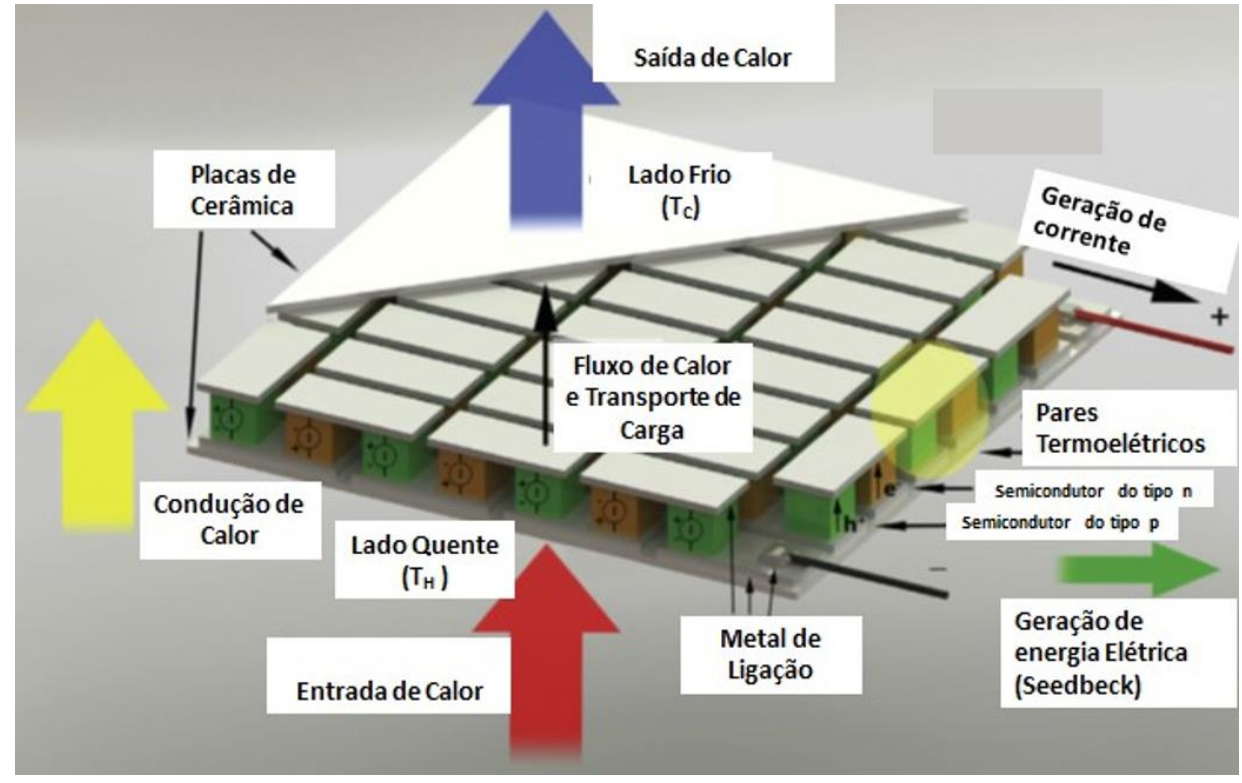

Figura 1: Modelo em 3D de um TEG e os principais efeitos físicos (adaptada de [5]).

Apesar da modelagem dos TEGs ser complexa, graças a não linearidade termoelétrica e corrente elétricas desconhecidas (em circuito fechado), além de mudanças nas condições de operações, como por exemplo, alterações de temperatura e de carga, estudos mostram que a simulação é possível, uma vez que os dados obtidos por modelos teóricos têm mostrados grande precisão quando comparados com dados experimentais [2,7-8].

Certos de que a recuperação de calor será uma das abordagens que irá desempenhar um papel importante no futuro, a JFE Steel Corporation (JFE), em 2012, juntamente com a Kelk Ldt. (Keltk), incorporou em uma linha de lingotamento contínuo, da própria JFE, um sistema de geração termelétrica. Esta incorporação foi realizada, e por meio de simulações matemáticas e comparação com dados experimentais concluiu-se que é possível recuperar cerca de $9 \mathrm{~kW}$ com a montagem desse sistema. Este resultado foi considerado promissor, uma vez que esta recuperação foi quase dez vezes maior que a fornecida por sistemas de energia solar, quan- 
do considerado um mesmo tamanho de coletor [2,9].

Tendo em vista o ambiente propício para a utilização de TEG na recuperação de energia residual envolvida nos processos da fabricação de aço, o presente trabalho tem como objetivo estimar, por meio de simulação matemática e métodos de ajuste - apoiados em revisão bibliográfica tanto de lingotamento contínuo quanto de equipamentos TEG - a quantidade de energia elétrica que um módulo elementar de TEG é capaz de gerar quando interage com uma placa quente de aço 1045, produto final de lingotamento contínuo, após etapas de desempeno e corte, e assim verificar, através de uma generalização dos resultados deste estudo, se a aplicação de TEG nesta situação específica, é realmente possível.

\section{MATERIAIS E MÉTODOS}

\subsection{Materiais}

O módulo TEG escolhido foi o CMO-32-62s fabricado pela TECTEG MFR [10]. Ele é composto de 32 pares termoelétricos de junção P-N e de uma chapa coletora, que pode ser feita de aço inoxidável AISI 304. Para a generalização dos resultados foram utilizados 1250 módulos TEG ligados em série, formando assim um conjunto TEG.

As velocidades de processo do lingotamento contínuo foram definidas como sendo $0,5 \mathrm{~m} / \mathrm{min}$ ou $0,3 \mathrm{~m} / \mathrm{min}$, e o coeficiente de transferência de calor entre a placa quente e a chapa coletora, foi definido em $0,180 \mathrm{~kW}^{0} \mathrm{C}^{-1} \mathrm{~m}^{-2}[11]$.

O comprimento e a largura são iguais tanto para a placa quente quanto para a chapa coletora, sendo de $3225 \mathrm{~mm}$ e 1612,5 mm respectivamente. Porém as espessuras da placa quente podem ser adotadas como sendo de $200 \mathrm{~mm}$ ou $400 \mathrm{~mm}$, enquanto a espessura da chapa coletora é de $4 \mathrm{~mm}$.

Para cálculo dos perfis teóricos de temperatura vs. tempo $(\mathrm{T}(\mathrm{t}))$ ao longo da chapa coletora e da placa quente, foi utilizado o software Deform ${ }^{T M}$-3D. Já para o processamento desses dados foram utilizados os softwares OriginPro 8, Excel 2013 e um software de apoio para realizar ajuste de curva.

\subsection{Métodos}

Ao final do processo de lingotamento contínuo, após o corte por oxicorte, a placa quente (denominada "PlacaQ"), produto do lingotamento contínuo, que está a $950^{\circ} \mathrm{C}$ ou $1050^{\circ} \mathrm{C}$, entra em contato com a chapa coletora (lado quente) do TEG, que se encontra a $35^{\circ} \mathrm{C}$. Estas temperaturas são aquelas tipicamente encontradas nesse tipo de processo [11-12]. Assim sendo, esses dois corpos buscarão o equilíbrio térmico - enquanto a placa quente resfria, a chapa coletora aquece. A temperatura da chapa coletora (denominada "ChapaC") em função do tempo, $\mathrm{T}(\mathrm{t})$, é de fundamental importância para fins de cálculo da potência gerada, uma vez que a potência gerada é função da diferença de temperatura, $\mathrm{P}(\mathrm{T})$, entre o lado frio e o lado quente do TEG.

Todas as dimensões, condições, temperaturas e propriedades do conjunto placa quente/chapa coletora foram inseridas no software Deform. Os dados de entrada para estas simulações são apresentados na Tabela 1. Dessa forma, foram gerados os perfis térmicos $\mathrm{T}(\mathrm{t})$ calculados pelo Deform. Por meio do software OriginPro e com base nos dados do fabricante foi plotada uma curva da potência em função da temperatura $[P(T)]$.

A partir das curvas geradas anteriormente foi possível obter uma curva da variação de potência em função do tempo $[\mathrm{P}(\mathrm{t})]$. Isso foi estabelecido por meio do ajuste de função composta $\mathrm{P}[\mathrm{T}(\mathrm{t})]$. Para se obter a função composta $\mathrm{P}[\mathrm{T}(\mathrm{t})]$, foi utilizado um software que, por meio de ajuste de curvas, determinou as equações que descreviam as curvas $\mathrm{P}(\mathrm{T})$ e $\mathrm{T}(\mathrm{t})$.

A etapa seguinte foi integrar a função resultante $\mathrm{P}(\mathrm{t})$, o que permite estimar a quantidade de energia gerada em um determinado intervalo de tempo $(\Delta t)$ para um módulo TEG. Para calcular a quantidade de energia elétrica gerada pelo módulo TEG analisado, é necessário definir o tempo de interação do TEG com a placa quente, uma vez que energia $(\mathrm{J})$ é resultado da potência $(\mathrm{J} / \mathrm{s})$ multiplicada por um intervalo de tempo (s).

Foram propostos dois esquemas separados de montagem, os quais foram associados ao final do processo de lingotamento contínuo (corte por oxicorte) para geração de energia elétrica. São estes:

- Projeto Esteira: com comprimento e largura iguais a 3225,0 mm e $1612,5 \mathrm{~mm}$, respectivamente, e módulos TEG cobrindo sua superfície externa, esta esteira deve ser posicionada após o corte por oxicorte, recebendo, portanto, placas quentes de aço. A velocidade de deslocamento da esteira deve ser igual à velocidade do processo de lingotamento contínuo, $0,5 \mathrm{~m} / \mathrm{min}$ ou $0,3 \mathrm{~m} / \mathrm{min}$, adotadas neste trabalho. O comprimento total de geração de energia para cada placa quente em contato com as chapas coletoras, chapas do tamanho de uma unidade TEG, será de pelo menos $6450,0 \mathrm{~mm}$, não considerando as regiões curvas do equipamento. Ou seja, 3225,0 mm em condição de aquecimento (entrada do produto na esteira) e 3225,0 mm em condição 
de resfriamento (parte inferior da esteira). Logo os tempos de interação para esta configuração foram 387s e $645 \mathrm{~s}$.

- Projeto Estante: com comprimento e largura iguais a $3225,0 \mathrm{~mm}$ e $1612,5 \mathrm{~mm}$ respectivamente, esta estante é fixa, sem movimento, com superfície recoberta pelos módulos TEG. Recebe as placas quentes advindas da etapa de oxicorte. Os tempos de interação calculados com base nas velocidades de lingotamento citadas anteriormente para esta configuração foram $327 \mathrm{~s}$ e $575 \mathrm{~s}$, descontados $60 \mathrm{~s}$ para posicionamento das placas, em aquecimento (EA), e 60s em resfriamento (R).

Tabela 1: Dados da entrada para controle de simulação feita pelo Deform.

\begin{tabular}{l|l}
\hline PRINCIPAL & SI \\
\hline Sistema de unidades & Lagrangeano incremental \\
\hline Tipo de simulação & Transferência de calor \\
\hline Modo de simulação & \\
\hline CONTROLE DE PROCESSO & 300 \\
\hline Número de passos da simulação & 1 \\
\hline Incremento para salvar passos & $3 \mathrm{~s}$ \\
\hline Incremento de tempo & \\
\hline ITERAÇÃO & Iteração direta \\
\hline Método de iteração & Esparso \\
\hline Solucionar temperatura & \\
\hline CONDIÇÃO DE PROCESSO & $35^{0} \mathrm{C}$ \\
\hline Temperatura ambiente & $0,02 \mathrm{~kW}^{0} \mathrm{C}^{-1} \mathrm{~m}^{-2}$ \\
\hline Coeficiente de convecção &
\end{tabular}

Foram realizadas 9 simulações, cujos os dados de entrada referentes a descrição dos objetos estão mostrados na Tabela 2, onde as identificações A (1-4) referem-se a etapa de aquecimento nas condições 1 a 4 (variando temperatura e espessura); R (1-2) ao resfriamento nas condições 1 e 2 (tempos de interação distintos); EA (1-2) ao aquecimento estante nas condições 1 e 2 . Note que o comprimento (3225,0 mm) e largura $(1612,5 \mathrm{~mm})$ da ChapaC e da PlacaQ foram omitidos da Tabela 2, uma vez que são sempre mantidos constantes para todas as condições.

A Figura 2 é um exemplo típico de configuração adotada para simulação dos campos térmicos (simulação A4 da Tabela 2). Podem ser visualizadas tanto a interface gráfica (Tela do software) quanto os resultados correspondentes ao passo de cálculo (Step) 129.

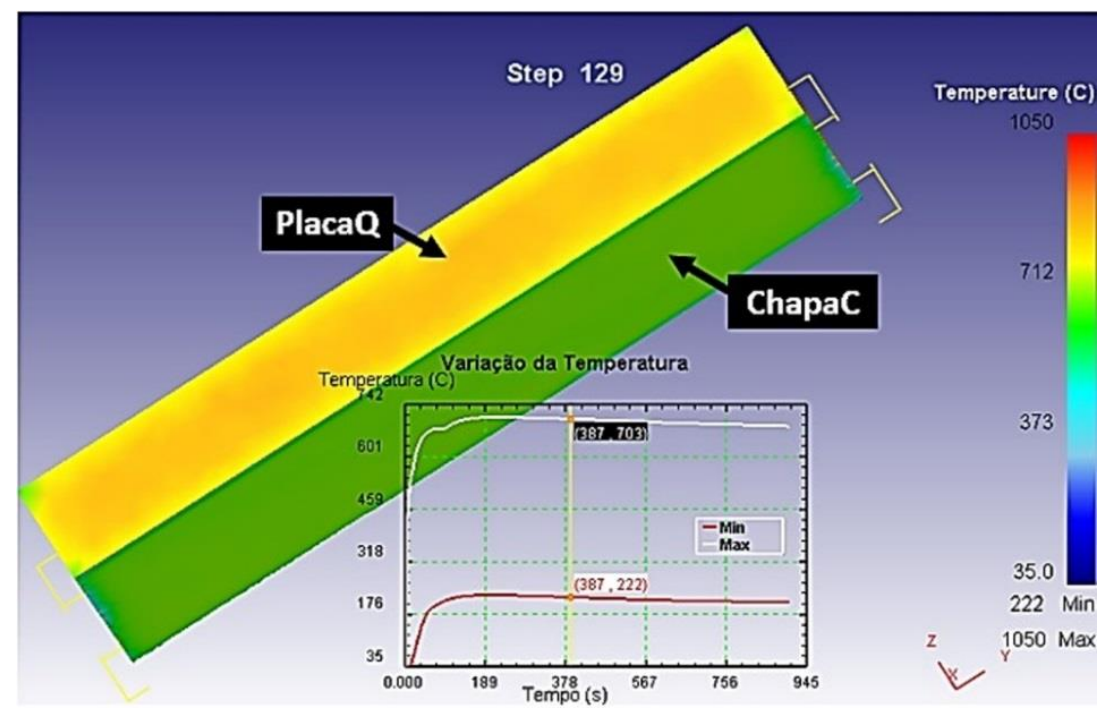

Figura 2: Exemplo de apresentação dos resultados no pós-processador do software Deform para cálculo de perfil térmico da PlacaQ do aço 1045 em contato com a ChapaC de aço inoxidável AISI 304.

Tabela 2: Dados de entrada referentes à descrição dos objetos simulados pelo Deform. 


\begin{tabular}{|c|c|c|c|c|c|}
\hline NOME DA SIMULAÇÃO & $\begin{array}{l}\text { OBJETOS ENVOLVI- } \\
\text { DOS }\end{array}$ & $\begin{array}{l}\text { ESPESSURA } \\
(\mathrm{mm})\end{array}$ & $\mathrm{T}_{\text {INICIALL }}\left({ }^{0} \mathrm{C}\right)$ & \begin{tabular}{|l|} 
MALHA \\
(MESH)
\end{tabular} & AÇO \\
\hline \multirow[b]{2}{*}{ A1 } & ChapaC & 4 & 35 & 300000 & AISI 304 \\
\hline & PlacaQ & 200 & 950 & 32000 & ASTM 1045 \\
\hline \multirow[b]{2}{*}{$\mathrm{A} 2$} & ChapaC & 4 & 35 & 300000 & AISI 304 \\
\hline & PlacaQ & 400 & 950 & 32000 & ASTM 1045 \\
\hline \multirow[b]{2}{*}{ A3 } & ChapaC & 4 & 35 & 300000 & AISI 304 \\
\hline & PlacaQ & 200 & 1050 & 32000 & ASTM 1045 \\
\hline \multirow[b]{2}{*}{ A4 } & ChapaC & 4 & 35 & 300000 & AISI 304 \\
\hline & PlacaQ & 400 & 1050 & 32000 & ASTM 1045 \\
\hline $\mathrm{R} 1$ & ChapaC & 4 & 647,5 & 300000 & AISI 304 \\
\hline R2 & ChapaC & 4 & 696,5 & 300000 & AISI 304 \\
\hline \multirow[b]{2}{*}{ EA1 } & ChapaC & 4 & 520 & 300000 & AISI 304 \\
\hline & PlacaQ & 200 & 950 & 32000 & ASTM 1045 \\
\hline \multirow[b]{2}{*}{ EA2 } & ChapaC & 4 & 560 & 300000 & AISI 304 \\
\hline & PlacaQ & 400 & 1045 & 32000 & ASTM 1045 \\
\hline
\end{tabular}

\section{RESULTADOS E DISCUSSÃO}

A Figura 3 mostra uma comparação entre a energia elétrica gerada nas etapas de aquecimento para um módulo TEG.

Nota-se que a simulação A4 gerou mais energia em função de apresentar maiores valores de espessura e temperatura inicial. Além disso, comparando as situações A1 com A2, e A3 com A4, observa-se que a mudança na espessura da PlacaQ exerce pouquíssima influência no processo de geração de energia, enquanto a temperatura inicial da mesma exerce uma forte influência, como o observado nas comparações entre A1 com A3 e A2 com A4. Porém o fator de maior impacto na geração de energia é o tempo de interação.

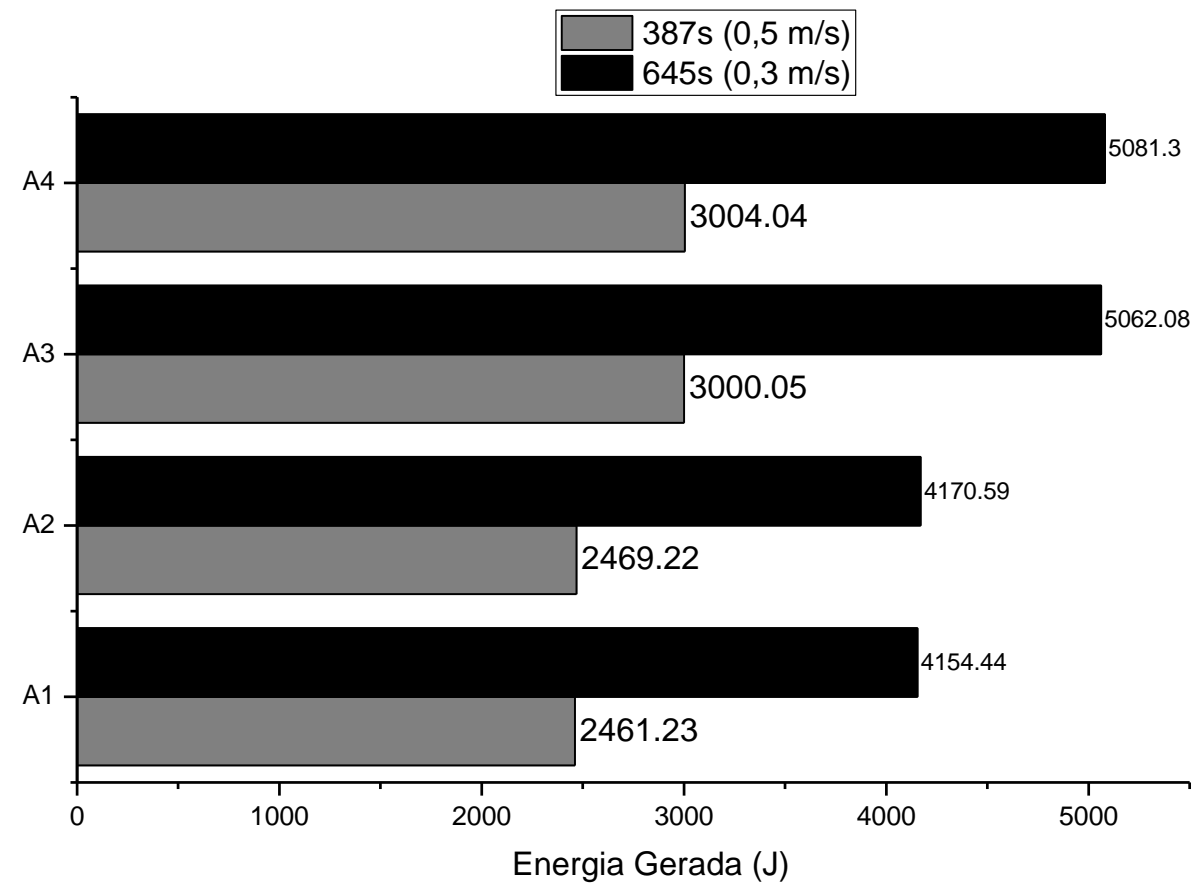

Figura 3: Comparação entre a energia elétrica gerada para as simulações A (1-4). Onde a temperatura e espessura da PlacaQ são, respectivamente: A1 - 950 ${ }^{\circ} \mathrm{C}$ e $200 \mathrm{~mm}$; A2 - 950 $\mathrm{C}$ e $400 \mathrm{~mm}$; A3 - $1050^{\circ} \mathrm{C}$ e $200 \mathrm{~mm}$ e A4 - $1050^{\circ} \mathrm{C}$ e $400 \mathrm{~mm}$.

Para efeito de comparação e para incorporar todas as etapas que envolvem um processo de reaproveitamento de energia (aquecimento e resfriamento) foram definidas a condição 1 (C1) como sendo aquela onde a PlacaQ refere-se a temperatura inicial e espessura iguais à $950^{\circ} \mathrm{C}$ e $200 \mathrm{~mm}$, respectivamente; e a condição 
2 (C2) como sendo aquela onde temos a PlacaQ com temperatura inicial e espessura iguais à $1050^{\circ} \mathrm{C} \mathrm{e}$ 400mm. Logo serão considerados apenas a melhor, simulação A4, e a pior situação, simulação A1. Além disso foi considerado o período de $12 \mathrm{~h}$ como uma jornada de produção diária de uma máquina de lingotamento contínuo. Assim temos que a geração de energia elétrica é dada pelas expressões (1) ou (2), para o projeto estante, e pelas expressões (3) e (4) para o projeto esteira, a depender da condição escolhida:

$$
\begin{aligned}
& \text { EEGestanteC1 }=1250\left[\mathrm{~A} 1_{\Delta \mathrm{t}}+\mathrm{nR} 1_{60}+\mathrm{nEA} 1_{\Delta \mathrm{t}-60}\right] \\
& \text { EEGestanteC2 }=1250\left[\mathrm{~A} 4_{\Delta \mathrm{t}}+\mathrm{nR} 260+\mathrm{nEA} 2_{\Delta \mathrm{t}-60}\right] \\
& \text { EEGesteiraC1 }=1250\left[(\mathrm{n}+1) \mathrm{A} 1_{\Delta \mathrm{t}}+\mathrm{nR} 1_{\Delta \mathrm{t}}\right] \\
& \text { EEGesteiraC2 }=1250\left[(\mathrm{n}+1) \mathrm{A} 4_{\Delta \mathrm{t}}+\mathrm{nR} 2_{\Delta \mathrm{t}}\right]
\end{aligned}
$$

onde $\Delta \mathrm{t}$ é $387 \mathrm{~s}$ ou $645 \mathrm{~s}$ e $\mathrm{n}=110(111-1)$ ou 66 (67-1) para as velocidades de $0,5 \mathrm{~m} / \mathrm{min}$ e $0,3 \mathrm{~m} / \mathrm{min}$, respectivamente. "n" é o número de vezes que esta simulação ocorre para um período de aproximadamente 12 horas.

As Figuras 4 e 5 são representações gráficas da quantidade de energia elétrica para os projetos "esteira” e "estante" com base nas equações acima. As representações referem-se ao total gerado no período de 12 horas e, uma vez que 1 Watt (W) é 1 Joule (J) por segundo (s), também é representada a potência média.

Para ambos os projetos é observado um aumento de aproximadamente $17 \%$ na quantidade de energia elétrica gerada, apenas mudando de $\mathrm{C} 1$ para C2.

Apesar do projeto "esteira" apresentar maior recuperação de energia no processo de lingotamento contínuo de aços, ele necessita de pelo menos duas vezes mais unidades TEG para ser confeccionado. Portanto, mesmo considerando que a recuperação do projeto "esteira" é aproximadamente $18 \%$ maior que a do projeto "estante", este último parece ser mais viável, tendo em vista que apresenta uma melhor relação custo/benefício.

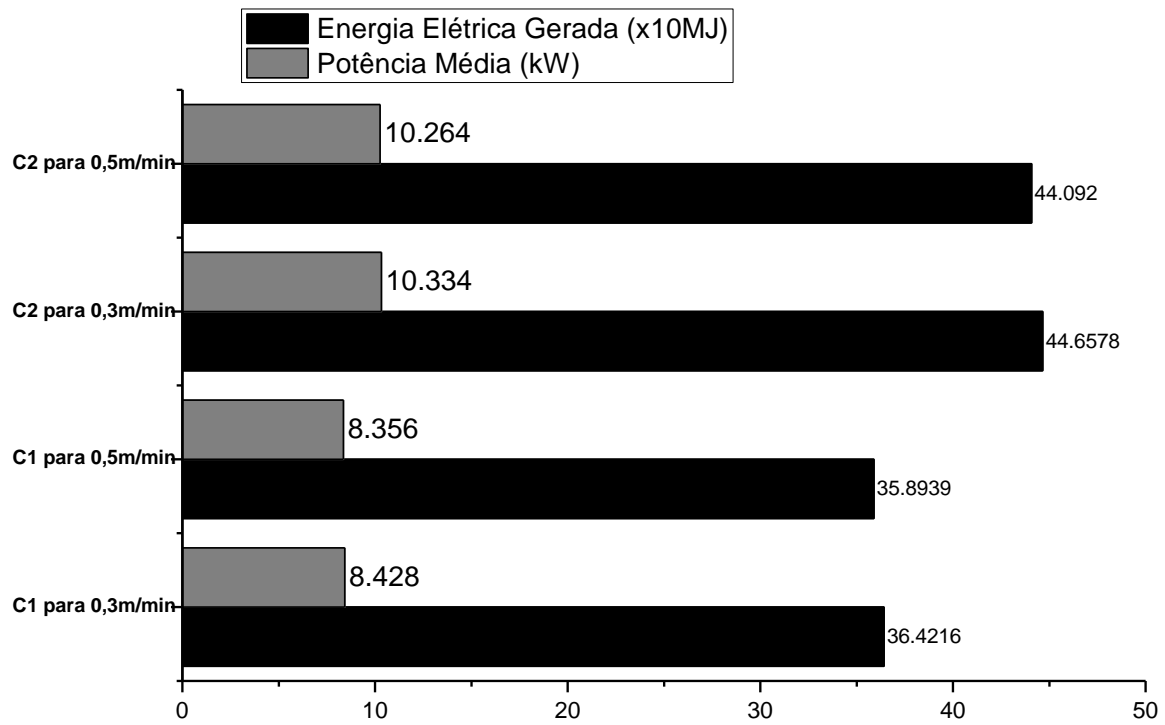

Figura 4: Potência média e energia elétrica média gerada no período de $12 \mathrm{~h}$ para as condições $\mathrm{C} 1$ (PlacaQ $=950{ }^{\circ} \mathrm{C}$ e $200 \mathrm{~mm})$ e $\mathrm{C} 2\left(\mathrm{PlacaQ}=1050^{\circ} \mathrm{C}\right.$ e $\left.400 \mathrm{~mm}\right)$ para o projeto 'estante'.

Considerando o melhor valor de potência média (melhor situação observada=10,33kW) para o projeto estante, e sabendo que o consumo médio de energia elétrica por uma residência brasileira foi de 157 kWh/mês no ano de 2017 [13], é possível, com esse sistema, fornecer energia elétrica para aproximadamente 23 residências. 


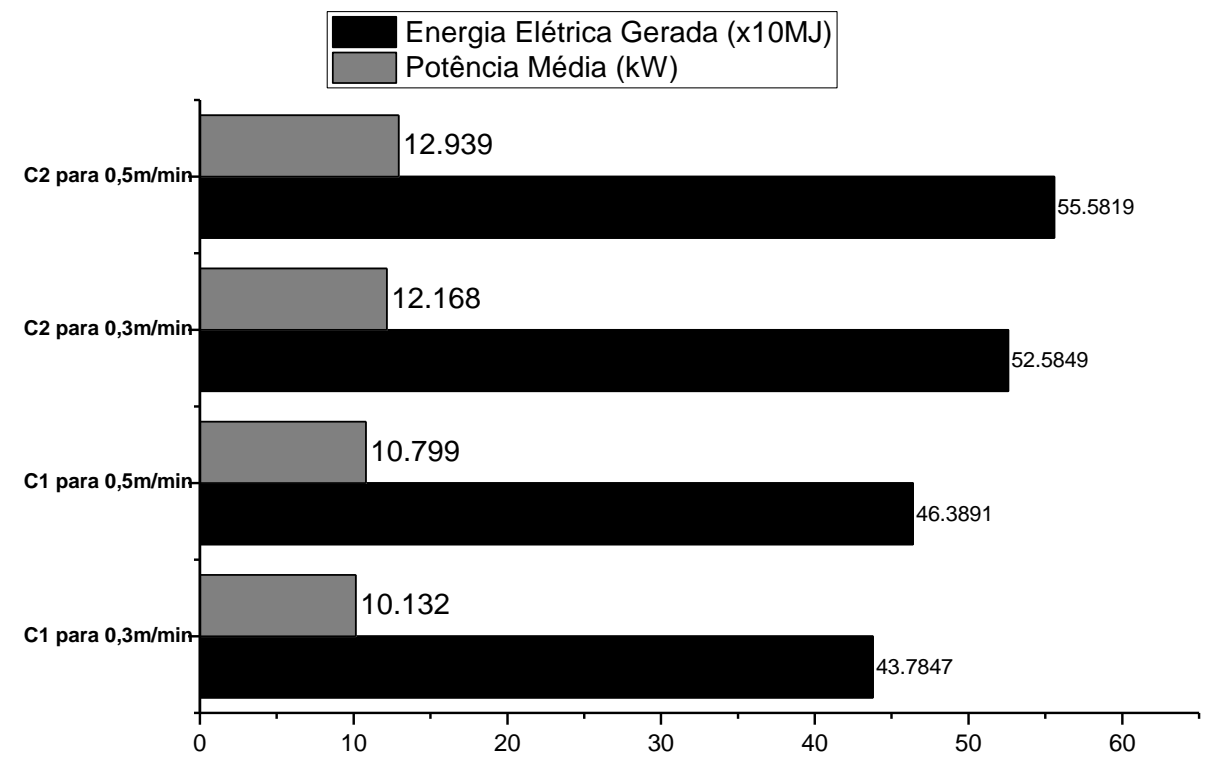

Figura 5: Potência média e energia elétrica média gerada no período de $12 \mathrm{~h}$ para as condições $\mathrm{C} 1\left(\mathrm{PlacaQ}=950{ }^{0} \mathrm{C}\right.$ e $200 \mathrm{~mm})$ e $\mathrm{C} 2\left(\mathrm{PlacaQ}=1050^{\circ} \mathrm{C}\right.$ e $\left.400 \mathrm{~mm}\right)$ para o projeto 'esteira'.

\section{CONCLUSÕES}

Os métodos de cálculos de energia gerada, tanto para unidades TEG quanto para o conjunto TEG, são eficazes, uma vez que os resultados obtidos apresentam boa concordância com a literatura.

Com base nas observações, concluiu-se que enquanto a variação da espessura da PlacaQ exerce pouca influência na geração de energia elétrica, a temperatura inicial da mesma e o tempo de interação (entre a PlacaQ e a ChapaC) exercem grande influência. Além disso, aparentemente há uma versatilidade nos projetos propostos quanto à velocidade de processo do lingotamento contínuo, uma vez que para um mesmo intervalo de tempo a geração se mostrou aproximadamente constante. Por fim, o "projeto estante", apesar de gerar uma quantidade de energia elétrica menor que o "projeto esteira", é o que apresenta melhor relação custo benefício.

\section{AGRADECIMENTOS}

Os autores agradecem ao apoio concedido pelo Departamento de Engenharia de Materiais (DEMa) da Universidade Federal de São Carlos (UFSCar) na realização desta pesquisa. Além disso, gostaríamos de agradecer as contribuições técnicas prestadas pelo Prof. Dr. Oscar Balancin.

\section{BIBLIOGRAFIA}

[1] IINO, Y., SOMA, F., HASHIMOTO, K. "Environmental Technologies in Steel Works", JFE Technical Report,Tokyo, n. 8, pp. 7-16, oct. 2006.

[2] KUROKI, T., KABEYA, K., MAKINO, K., et al., "Thermoelectric Generation Using Waste Heat in Steel Works", Journal of Eletronic Materials, v. 43, n. 6, pp. 2405-2410, 2014.

[3] LEBLANC, S. "Thermoelectric Generators: Linking Material Properties and Systems Engineering for Waste Heat Recovery Applications”, Sustainable Materials and Technologies, v. 1-2, pp. 26-35. 2014.

[4] CUENT, A. Nanostructured Thermoelectrics, http://www.npl.co.uk/sciencetechnology/electrochemistry/research/nanostructured-thermoelectrics. Acesso em 6 de maio de 2016.

[5] DU, C.Y., WEN, C.D. "Experimental Investigation and Numerical Analysis for one-stage Thermoelectric Cooler Considering Thomson Effect”, International J Heat Mass Transfer, v.54, pp. 4875-4884. 2011.

[6] ROWE, D.M. Miniature Semiconductor Thermoelectric Devices, Handbook of Thermoelectric. Boca Raton: CRC Press, 1995.

[7] MONTECUCCO, A., KNOX, A.K. "Accurate Simulation of Thermoelectric Power Generating Systems", Applied Energy, v. 118, pp. 166-172. 2014. 
[8] RABARI, R., MAHMUD, S., DUTTA, A. "Numerical Simulation of Nanostructured Thermoelectric Generator Considering Surface to Surrounding Convection", International Communications in Heat and Mass Transfer, v. 56, pp. 146-151, 2014.

[9] KUROKI, T., MURAI, R., MAKINO, K., et al., "Research and Development for Thermoelectric Generation Technology Using Waste Heat from Steelmaking Process", Journal of Eletronic Materials, v. 44, n. 6, p. 2405-2410, 2015.

[10] TECTEG MFR, Product, <http://tecteg.com/product/cmo-32-62s/>. Acesso em 11 de maio de 2016.

[11] GARCIA, A., SPIM, J. A., SANTOS, C. A., et al., Lingotamento Contínuo de Aços, 1 ed, São Paulo, Associação Brasileira de Metalurgia e Materiais, 2006.

[12] RIZZO, E.M.S. Introdução aos Processos Siderúrgicos. São Paulo, Associação Brasileira de Metalúrgia e Materiais, 2005.

[13] Editoria, OPOVO ONLINE, https://www.opovo.com.br/noticias/economia/ae/2018/02/consumo-deenergia-cresce-0-8-em-2017-primeira-alta-em-tres-anos.html. Acessado em abril de 2018.

\section{ORCID}

Rafael Formenton Macedo dos Santos

José Eduardo Spinelli

https://orcid.org/0000-0003-1710-487X

https://orcid.org/0000-0003-0611-1038 